\title{
Correlations between Left Ventricular and Left Atrial Function Assessed by Speckle Tracking Echocardiography in Patients with Treated Well-Controlled Arterial Hypertension
}

\author{
Beata Uziębło-̇̇yczkowska (iD) and Paweł Krzesiński \\ Department of Cardiology and Internal Diseases, Military Institute of Medicine, Warsaw, Poland \\ Correspondence should be addressed to Beata Uziębło-Życzkowska; buzieblo-zyczkowska@wim.mil.pl
}

Received 14 November 2020; Revised 24 March 2021; Accepted 31 March 2021; Published 12 April 2021

Academic Editor: Robert Chen

Copyright (C) 2021 Beata Uziębło-Życzkowska and Paweł Krzesiński. This is an open access article distributed under the Creative Commons Attribution License, which permits unrestricted use, distribution, and reproduction in any medium, provided the original work is properly cited.

\begin{abstract}
Introduction. Even in patients with well-controlled arterial hypertension (AH) and without significant comorbidities, left ventricular (LV) and left atrial (LA) strain abnormalities may sometimes be found in speckle-tracking echocardiography. Therefore, the aim of this study was to investigate the correlation between LA strain and LV diastolic and systolic function in a group of patients with treated, well-controlled AH. Material and methods. LA contractile, conduit, and reservoir function, together with echocardiographic signs of LV diastolic function and LV global longitudinal strain (LV GLS), were assessed in 101 patients with treated, well-controlled $\mathrm{AH}$ who met the standard criteria of normal LV ejection fraction (LVEF) and normal LV diastolic function. Results. A relevant percentage of study participants presented lower than reference LV ( $-18.7 \%$ for LV GLS) and LA strain (32.9\% for LAS during reservoir phase, LASr; $-15.9 \%$ for LAS during contraction phase, LASct; and $-13.9 \%$ for LAS during conduit phase, LAScd) values. Moreover, there were statistically significant differences in LA longitudinal strain (LAS) values (LASr (31.43 vs. 36.33; $p=0.0007)$ and LAS LAScd ( -13.09 vs. $-15.79 ; p=0.008)$ ) between patients with high (>the absolute value of $-20 \%$ ) and lower ( $\leq$ the absolute value of $-20 \%$ ) LV GLS, confirmed by significant correlations between LASr, LAScd, and GLS. In the correlations analysis between LAS values and LV diastolic function parameters, statistical significance was obtained for the following: LASct (contraction) versus e'avg, LASct versus E/A, LASct versus A, LAScd versus e'avg, LAScd versus E/A, and LAScd versus A. Conclusions. LV and LA strain abnormalities occurred within a significant percentage of patients with treated, well-controlled AH. Impaired LA strain is associated with lower LV strain and reduced LV diastolic function parameters, reflecting both the passive and active properties of the LA.
\end{abstract}

\section{Introduction}

Arterial hypertension (AH) is one of the most prevalent cardiovascular diseases. European guidelines present strong evidence for the use of antihypertensive treatment in moderate and severe $\mathrm{AH}$, but the usage of pharmacotherapy in patients with mild $\mathrm{AH}$ is still debated [1]. Especially in young people, marginally elevated blood pressure (BP) is thought to have no significant clinical consequences. However, the results of the Systolic Blood Pressure Intervention Trial (SPRINT) [2], which forced the American cardiology societies to decrease BP thresholds for diagnosing
AH [3], sparked a discussion on cardiovascular risk in mild $\mathrm{AH}$.

Even in patients with well-controlled AH, without significant comorbidities and with normal echocardiographic indices of left ventricular (LV) systolic and diastolic dysfunction, LV and left atrial (LA) strain abnormalities may sometimes be found in speckle-tracking echocardiography (STE) [4-6]. In our earlier study, we revealed that, in hypertensive patients with normal LV ejection fraction (EF), left ventricular global longitudinal strain (LV GLS) is reduced compared to healthy people, and its value improves after effective antihypertensive treatment [4]. Also, for left 
atrial (LA) function, recent studies using STE showed that in hypertensives, LA strain parameters might be impaired, despite normal LA size [7, 8].

Based on these reports, we hypothesized that even in the early stage of $\mathrm{AH}$, there might be some interrelated subclinical LV and LA abnormalities, detectable by STE. Therefore, we decided to investigate the relation between LA strain and LV diastolic and systolic function in a group of patients with treated, well-controlled $\mathrm{AH}$.

\section{Material and Methods}

2.1. Study Group. It was a retrospective, secondary analysis of the data collected in the Noninvasive Haemodynamic Assessment in Hypertension (FINE-PATH) study (ClinicalTrials.gov Identifier NCT01996085), which was conducted in the period 2011-2014 [9]. In brief, this trial had a prospective, randomized, controlled design (144 patients enrolled) to assess a novel approach to the treatment of $\mathrm{AH}$; the study involved patients with at least a three-month history of AH defined according to the European Society of Cardiology guidelines [1]. The exclusion criteria included secondary $\mathrm{AH}$, chronic kidney disease, systolic heart failure, cardiomyopathy, significant arrhythmias, significant valvular heart disease, chronic obstructive pulmonary disease, previously diagnosed diabetes mellitus, polyneuropathy, and peripheral vascular disease. The following drug classes were used: beta-blockers, angiotensin-converting enzyme inhibitors, angiotensin receptor blockers, calcium channel blockers, and diuretics, either alone or in combination.

In this secondary analysis from among the whole group who attended a controlled visit after 12 months of treatment $(n=121), 108$ patients with acceptable ultrasound image quality were selected. In the final analysis, 101 patients with normal LV diastolic function evaluated based on current guidelines [10] were included. Collected data included demographic characteristics, the results of a complete clinical examination, $24 \mathrm{~h}$ ambulatory blood pressure (ABP) monitoring (ABPM), antihypertensive treatment, and transthoracic echocardiography.

2.2. Ambulatory Blood Pressure Monitoring. Ambulatory blood pressure monitoring (Spacelabs 90207, Spacelabs, Medical Inc, Redmond, USA) was performed within 2 weeks before echocardiography. The time from 6 a.m. to 10 p.m. was considered a daily activity period (daytime) with automatic blood pressure measurement in 10-minute intervals. During night rest (night-time: 10 p.m.-6 a.m.) the measurement was performed every 30 minutes. As a good blood pressure control (well-treated hypertension) was considered a mean 24-hour systolic $\mathrm{BP}<130 \mathrm{mmHg}$ and diastolic $\mathrm{BP}<80 \mathrm{mmHg}$, a daytime systolic $\mathrm{BP}<135 \mathrm{mmHg}$ and diastolic $\mathrm{BP}<85 \mathrm{mmHg}$, and night-time systolic $\mathrm{BP}<120 \mathrm{mmHg}$ and diastolic $\mathrm{BP}<70 \mathrm{mmHg}$.

\subsection{Standard Transthoracic Echocardiography.} Transthoracic echocardiography was performed using a high-quality echocardiograph (Vivid 7 or E95, General Electric, United States). The examinations were analyzed offline by an experienced echocardiographer accredited by the Section of Echocardiography of the Polish Cardiac Society, echocardiography laboratory. All LV and LA measurements were made according to the current guidelines of the European Society of Cardiology [11]. To estimate the size and function of the LA, the following standard parameters were measured: LA end-diastolic diameter, LA area, LA volume (LAV), and LA indexed volume (LAVI). LAV and LAVI were measured using a biplane algorithm from the apical four-chamber (A4C) and two-chamber (A2C) views. LA enlargement was defined as LAVI $>34 \mathrm{ml} / \mathrm{m}^{2}$. To assess LV function, LVEF was calculated using the biplane Simpson formula. LV mass (LVM) was calculated using the linear method according to the recommendations for cardiac chamber quantification by echocardiography in adults [11]. Using the parasternal longitudinal axis view, the thicknesses of the interventricular septal, the inferolateral walls, and the LV end-diastolic and end-systolic diameters were obtained. To diagnose LV hypertrophy, LVM was indexed to the body surface area (BSA) and calculated using the DuBois formula (indexed LVM-LVMI). Left ventricular hypertrophy (LVH) was diagnosed as recommended (cut-off values for women are LVMI $>95 \mathrm{~g} / \mathrm{m}^{2}$ and for men, LVMI $>115 \mathrm{~g} / \mathrm{m}^{2}$ ).

Diagnosis of LV diastolic dysfunction was based on the current guidelines [10], where the parameters for its identification and their cutoffs are as follows: LAVI $>34 \mathrm{ml} / \mathrm{m} 2$, septal annular $e^{\prime}$ velocity $<7 \mathrm{~cm} / \mathrm{s}$, lateral annular e' velocity $<10 \mathrm{~cm} / \mathrm{s}$, average $E / e^{\prime}$ ratio $>14$, and peak tricuspid regurgitation velocity $>2.8 \mathrm{~m} / \mathrm{s}$. Waves $E$ and $A$ of the mitral inflow velocity by pulsed wave Doppler from the apical fourchamber view, the E/A ratio, and the velocity waves ( $e$ ' and $\left.a^{\prime}\right)$ of the mitral annulus septal and lateral regions were recorded using tissue Doppler imaging. An average value of septal and lateral mitral annulus velocities was used to estimate $E / e^{\prime}$ ratio.

2.4. Speckle-Tracking Echocardiography (STE). Regional and global longitudinal 2D LA and LV strain was analyzed by STE using GE EchoPAC BT 12 software. LV GLS was assessed using automated imaging software. Detection of the tracked area was performed semiautomatically with two points selected at the level of the mitral annulus and the third point at the apex with the possibility of manual adjustments. The LV GLS values were averaged for all 17 LV segments: seven in the apical four-chamber view, six in the apical twochamber view, and six in the apical three-chamber view.

Analysis of LA strain was performed offline, obtained from a nonforeshortened apical, both A4C- and A2C-view images, using conventional 2D gray-scale echocardiography. High frame rates (60-80 frames per second) were used for analysis as recommended in the Expert Consensus Statement [12]. The analysis was performed by an experienced echocardiographer using acoustic-tracking software (EchoPAC, General Electric, USA), allowing offline semiautomated analysis of speckle-based strain. The LA endocardial border was manually traced in both the A4C and A2C views. An epicardial border was automatically 


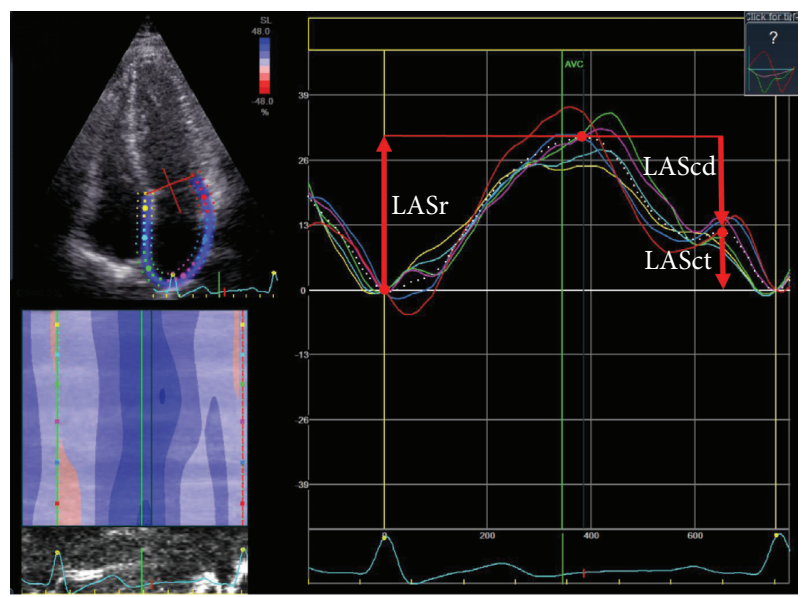

Figure 1: Measurements of left atrial strain parameters with the zero strain reference at the end-diastole.

generated by the software, creating a region of interest. The LA was contoured, extrapolating across the pulmonary veins and LA appendage orifice. Then, after eventual manual adjustment of the ROI shape, the software divided the region of interest into six segments and generated a longitudinal strain curve. To assess all LA strain values, the QRS wave onset was set as a reference point as recommended in the consensus document [13]. The obtained LA longitudinal strain (LAS) parameters were as follows [13]: positive peak strain during the reservoir phase (LASr), corresponding to the atrial reservoir function (positive value); next strain value during early diastole, corresponding to the atrial conduit function and identified as the LA-strain-duringconduit phase (LAScd-negative value), and the LA strain during late diastole, corresponding to active atrial contraction and identified as the LA-strain-during-contraction phase (LASct-negative value). LASr, LAScd, and LASct were calculated by averaging the values observed in all the LA segments (global LASr, LAScd, and LASct). When some segments were excluded due to the inability to achieve adequate tracking, LAS was calculated by averaging the values measured in the remaining segments. All measurements were obtained during sinus rhythm. As reference LA strain values, we adopted those given in a large meta-analysis carried out by Pathan et al., which included 2,542 healthy subjects [14]. Detailed measurements of LASr, LAScd, and LASct are presented in Figure 1.

To assess the intraobserver variability of LASr A4C, LASr A2C, LASct A4C, and LASct A2C, 20 patients were randomly selected. Intraobserver variability coefficients were calculated using images independently recorded at two different times by the same observer. The intraclass correlation coefficient together with the mean difference (95\% CI) of two measurements in Bland-Altman analysis, divided by the mean of those two measurements and given as percentages, were calculated for intraobserver variability. The repeatability of the LAS measurements was high. The intraclass correlation coefficients for the intraobserver variability of LASs were 0.99 for LASr A4C, 0.98 for LASr A2C, 0.99 for LASct A4C, and 0.98 for LASct A2C. The mean difference divided by the mean of two measurements for intraobserver variability was $0.4 \%(-1.1 \%-2,0 \%)$ for LASr $\mathrm{A} 4 \mathrm{C}, 0.6 \%(-1.6 \%-28 \%)$ for LASr A2C, $0.2 \%(-3.7 \%-6.6 \%)$ for LASct A4C, and $0.5 \%(-3.1 \%-4.1 \%)$ for LASct A2C.

2.5. Statistical Analysis. Statistical analyses were conducted with Statistica 12.0 (StatSoft Inc., Tulsa, OK, USA). The distribution and normality of data were assessed visually and with the Shapiro-Wilk test. Continuous variables were presented as the mean \pm standard deviation (SD), whereas categorical variables were presented as absolute and relative values (percentages). A comparison analysis was conducted for two subgroups: patients with High GLS (>the absolute value of $-20 \%$; "High GLS"; $n=30$ ) and Lower GLS ( $\leq$ the absolute value of $-20 \%$; "Lower GLS"; $n=71$ ). The cut-off value was in accordance with the current recommendations [11]. The Student's $t$-test was used for normally distributed data, whereas the Mann-Whitney $U$ test was used for data with nonnormal distribution. A $p$ value of $<0.05$ was considered statistically significant.

2.6. Ethical Consideration. The studies were conducted according to Good Clinical Practice guidelines and the Declaration of Helsinki. The study protocol was approved by the local ethics committee, and each patient provided written consent.

\section{Results}

3.1. Baseline Characteristic. A total of 101 patients (64 men with a mean age of 45.2 years) with well-controlled blood pressure and heart rate control were selected to participate in the study. The mean values of SBP and DBP obtained from ABPM for all patients were $119.7 \pm 9.2 \mathrm{~mm}$ $\mathrm{Hg}$ and $76.7 \pm 7.5 \mathrm{~mm} \mathrm{Hg}$, respectively. Details of their demographic, clinical, and treatment data are presented in Table 1.

3.2. Echocardiographic Assessment. The mean value of LAVI was $29.1(6.8) \mathrm{m}^{1} / \mathrm{m}^{2}$, and only $17(16.8 \%)$ patients fulfilled the criteria of LA enlargement. Myocardial hypertrophy was diagnosed in $14(13.9 \%)$ subjects, and the mean LVMI for the whole group was $89.5( \pm 17.5) \mathrm{g} / \mathrm{m}^{2}$. The study population was characterized by normal LVEF $(65.2 \pm 3.3 \%)$. However, the mean LV GLS $(-18.7 \pm 2.6 \%)$ was lower than the value $(-20 \%)$ which is called high in a healthy person according to ESC guidelines [1]. Also, the analysis of LAS revealed lower values than those previously reported as normal (39\% for LASr, $-17 \%$ for LASct, and $-23 \%$ for LAScd) [14]. In our group, these LAS parameters were $32.9 \%,-15.9 \%$, and $-13.9 \%$, respectively. Detailed echocardiographic data are summarized in Table 2.

3.3. Comparison of Subgroups with Respect to LV GLS. There were no differences between the subgroups "High GLS" and "Lower GLS" for age, sex distribution, or antihypertensive treatment. LA strain measurements were 
TABle 1: Demographic, clinical, and treatment data for the study population.

\begin{tabular}{lc}
\hline Variable & Study population, $n=101$ \\
\hline Demographic data & $45.2(10.2)$ \\
Age (years), mean (SD) & $64(63.4)$ \\
Male, $n$ (\%) & $66.9(8.5)$ \\
\hline Clinical data, mean (SD) & $119.9(9.8)$ \\
Heart rhythm (bpm), mean (SD) & $76.9(7.5)$ \\
24 h average systolic blood pressure, mean (SD) & $69(68.3)$ \\
24 h average diastolic blood pressure, mean (SD) & $12(11.9)$ \\
Antihypertensive treatment & $31(30.7)$ \\
Acei, $n(\%)$ & $23(22.8)$ \\
Arb, $n$ (\%) & $13(12.9)$ \\
Diuretics, $n(\%)$ & $48(47.5)$ \\
Beta-blocker, $n(\%)$ & $39(38.6)$ \\
Calcium channel blocker, $n(\%)$ & $8(7.9)$ \\
\hline Therapy & $6(5.9)$ \\
Monotherapy, $n(\%)$ & \\
Dual therapy, $n$ (\%) & \\
Triple therapy, $n(\%)$ & \\
Only nonpharmacological recomendations, $n(\%)$ & \\
\hline
\end{tabular}

ACEI, angiotensin-converting enzyme inhibitor; ARB, angiotensin receptor blocker

TABLE 2: Echocardiography data for the study population.

\begin{tabular}{lc}
\hline Variable & Study population, $n=101$ \\
\hline Standard echocardiography findings & \\
LAVI, $\mathrm{m}^{1} / \mathrm{m}^{2}$, mean (SD) & $29.1(6.8)$ \\
Enlargement LA,$n(\%)$ & $17(16.9 \%)$ \\
$E / A<0,8, n(\%)$ & $17(16.8)$ \\
$E$ avg $(\mathrm{cm} / \mathrm{s})$, mean (SD) & $10.9(2.3)$ \\
$E / e^{\prime}$ avg, mean (SD) & $6.7(1.6)$ \\
$E(\mathrm{~cm} / \mathrm{s})$, mean (SD) & $71.3(15.8)$ \\
$A(\mathrm{~cm} / \mathrm{s})$, mean (SD) & $62.3(13.2)$ \\
LVEF (\%), mean (SD) & $65.2(3.3)$ \\
E/A, mean (SD) & $1.19(0.35)$ \\
LVMI (g/m $\left.{ }^{2}\right)$, mean (SD) & $89.5(17.5)$ \\
LVH**, $n(\%)$ & $14(13.9)$ \\
\hline Speckle-tracking echocardiography findings & \\
LASr (\%), mean (SD) & $32.9(6.4)$ \\
LASct (\%), mean (SD) & $15.9(3.9)$ \\
LAScd (\%), mean (SD) & $13.9(4.7)$ \\
LV GLS (\%), mean (SD) & $18.7(2.6)$ \\
GLS $<20 \%, n$ (\%) & $71(67.3)$ \\
\hline
\end{tabular}

LA, left atrial; LAScd, left atrial strain conduit; LASct, left atrial strain contraction; LASr, left atrial strain reservoir; LAVI, left atrial volume index; LVEF, left ventricular ejection fraction; LV GLS, left ventricular global longitudinal strain; LVH, left ventricular hypertrophy; LVMI, left ventricular mass index. ${ }^{*}$ Enlargement LA defined as LAVI $>20 \mathrm{~m}^{1} / \mathrm{m}^{2}$. ${ }^{* *} \mathrm{LVH}$ defined as LVMI $>95 \mathrm{~g} / \mathrm{m}^{2}$ for females and LVMI $>115 \mathrm{~g} / \mathrm{m}^{2}$ for males.

significantly lower in patients with Lower GLS as compared to those with High GLS. Patients in the "Lower GLS" subgroup presented significantly reduced LASr $(p=0.0007)$ and LAScd $(p=0.008)$ as well as an increased $E / e^{\prime}$ ratio $(p=0.037)$. These results were supported by correlations with LV GLS for LASr, LAScd, E/e' ratio, and $E^{\prime}$ value. GLS also proved to be correlated with LVEF. Detailed comparative data are presented in Table 3 and graphic presentation for significant correlations is in Figure 2.
3.4. Correlation of Atrial Strain with LV Diastolic Function Parameters. In the correlation analysis of LAS values and LV diastolic function parameters, significant results were obtained for LASct versus $e^{\prime}$ avg $(p=0.002)$, LASct versus $E$ / $A(p<0.001)$, LASct versus A $(p=0.004)$, LAScd versus $e$ 'avg $(p=0.004)$, LAScd versus $E / A(p=0.001)$, and LAScd versus A $(p=0.001)$ (see Table 4$)$.

\section{Discussion}

Our results revealed that even in patients with treated wellcontrolled $\mathrm{AH}$, who have good $\mathrm{BP}$ control and no LV dysfunction in standard echocardiographic evaluation, the values of LV and LA strain measured by STE might be impaired. We also noted that LV GLS is the best correlated with LA strain. Surprisingly, its correlations with LASr and LAScd were even stronger than with LVEF. The interplay between LV diastolic pressures and LA function was also identified and confirmed in correlations of some LAS with $E$ / $A$ and $e^{\prime}$.

We intended to investigate a group of young, welltreated hypertensives, mostly free from heart function impairment in standard echocardiography (LVH confirmed in only 14\%). The exclusion of subjects with reduced and midrange LVEF from the FINEPATH study along with the additional criteria for this particular analysis (LV diastolic dysfunction) provided a set of data fulfilling these assumptions. Even the prevalence of myocardial hypertrophy was very low (13.9\%).

However, the use of novel echocardiographic parameters revealed a high frequency of subtle abnormalities occurring in this group. More than two-thirds of patients showed LV GLS values below $-20 \%$, and LAS values were also lower than reference values. Previous studies also reported that, in hypertensive patients, LV GLS is decreased despite the lack of other comorbidities [5,6]. In our earlier group of 125 patients with mild hypertension [5], we revealed impaired 
TABLE 3: The comparison between "High GLS" and the "Lower GLS" subgroups and correlations analysis between the LV GLS and other variables.

\begin{tabular}{|c|c|c|c|c|c|}
\hline & $\begin{array}{c}\text { LV Lower GLS } \\
\text { subgroup, mean (SD) }\end{array}$ & $\begin{array}{l}\text { LV high GLS subgroup, } \\
\text { mean (SD) }\end{array}$ & $\begin{array}{c}p \text { (for } \\
\text { comparison) }\end{array}$ & $\begin{array}{c}\text { Correlations LV GLS versus } \\
\text { variable }(R) \\
\end{array}$ & $\begin{array}{c}p(\text { for } \\
\text { correlations })\end{array}$ \\
\hline $\begin{array}{l}\text { Age, years, } \\
\text { mean (SD) }\end{array}$ & $44.86(10.02)$ & $46.13(10.70)$ & 0.57 & 0.13 & 0.18 \\
\hline Men/women, $n(\%)$ & $48(67.6)$ & $16(53.3)$ & 0.17 & - & - \\
\hline \multicolumn{6}{|c|}{ Echocardiography parameters, mean (SD) } \\
\hline$E^{\prime} \mathrm{m}(\mathrm{cm} / \mathrm{s})$ & $9.38(1.94)$ & $9.53(2.10)$ & 0.75 & -0.02 & 0.86 \\
\hline E'l $(\mathrm{cm} / \mathrm{s})$ & $12.56(3.55)$ & $12.23(2.14)$ & 0.62 & -0.06 & 0.57 \\
\hline E'avg $(\mathrm{cm} / \mathrm{s})$ & $10.97(2.43)$ & $10.88(1.89)$ & 0.86 & -0.02 & 0.60 \\
\hline$E / e^{\prime}$ avg & $6.48(1.60)$ & $7.10(1.51)$ & 0.04 & 0.23 & 0.02 \\
\hline $\mathrm{E}(\mathrm{cm} / \mathrm{s})$ & $69.42(16.30)$ & $75.77(13.89)$ & 0.06 & 0.25 & 0.012 \\
\hline $\mathrm{A}(\mathrm{cm} / \mathrm{s})$ & $61.82(13.32)$ & $63.60(13.00)$ & 0.54 & 0.19 & 0.06 \\
\hline $\mathrm{A}^{\prime} \mathrm{m}(\mathrm{cm} / \mathrm{s})$ & $9.82(1.55)$ & $9.53(1.31)$ & 0.39 & 0.04 & 0.70 \\
\hline$A^{\prime} l(\mathrm{~cm} / \mathrm{s})$ & $10.86(2.21)$ & $10.17(2.51)$ & 0.14 & 0.03 & 0.79 \\
\hline LVEF (\%) & $64.68(3.22)$ & $66.47(3.08)$ & 0.01 & 0.27 & 0.007 \\
\hline $\mathrm{E} / \mathrm{A}$ & $1.17(0.36)$ & $1.23(0.31)$ & 0.37 & 0.01 & 0.99 \\
\hline LVMI $\left(\mathrm{g} / \mathrm{m}^{2}\right)$ & $90.1(17.4)$ & $87.8(18.2)$ & 0.57 & -0.11 & 0.30 \\
\hline LAVI $\left(\mathrm{m}^{1} / \mathrm{m}^{2}\right)$ & $28.51(6.61)$ & $30.50(6.99)$ & 0.05 & 0.17 & 0.09 \\
\hline \multicolumn{6}{|c|}{$\begin{array}{l}\text { Speckle-tracking echocardiography parameters, mean (SD) } \\
\end{array}$} \\
\hline LASr (\%) & $31.43(5.5)$ & $36.33(7.20)$ & 0.0007 & 0.37 & $<0.001$ \\
\hline LASct $(\%)$ & $-15.80(3.96)$ & $-16.16(3.88)$ & 0.67 & 0.18 & 0.08 \\
\hline LAScd $(\%)$ & $-13.09(4.11)$ & $-15.79(5.42)$ & 0.008 & 0.29 & 0.003 \\
\hline LV GLS (\%) & $-17.40(1.73)$ & $-21.79(1.18)$ & $<0.0001$ & - & \\
\hline
\end{tabular}

The abbreviations are as in Table 2.

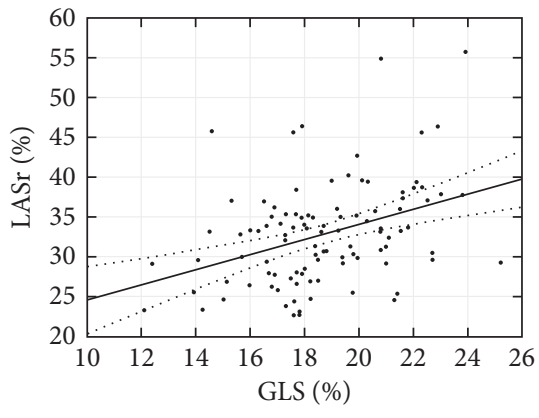

(a)

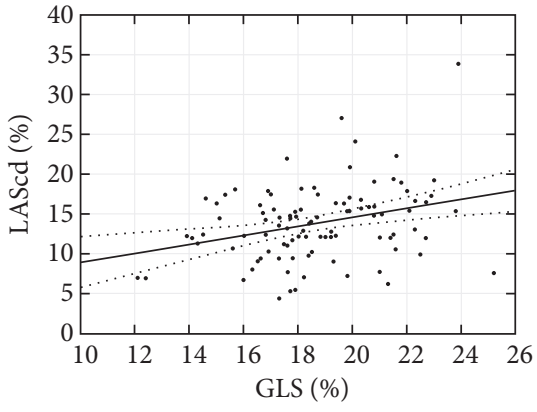

(b)

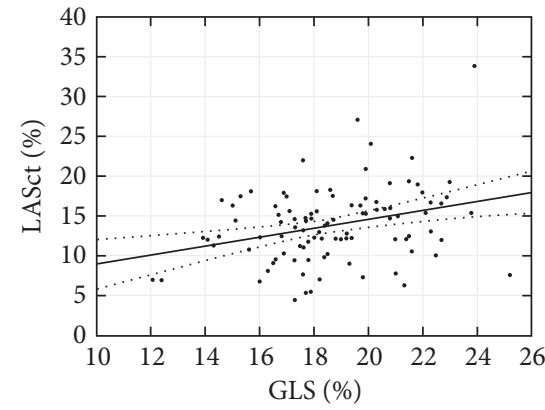

(c)

Figure 2: Correlations plots for GLS versus LASr $(R=0.37 ; p<0.001$, left chart), GLS versus LAScd $(R=0.29, p<0.01$; middle chart $)$ and GLS versus LASct $(R=0.18$, ns; right chart) (LAScd, left atrial strain conduit; LASct, left atrial strain contraction; LASr, left atrial strain reservoir; and GLS, left ventricular global longitudinal strain).

TABLE 4: Correlation of LAS values with LV diastolic function parameters.

\begin{tabular}{lccccc}
\hline & $E$ avg $(\mathrm{cm} / \mathrm{s})$ & $E / e^{\prime}$ avg & $E / A$ & $E(\mathrm{~cm} / \mathrm{s})$ & $A(\mathrm{~cm} / \mathrm{s})$ \\
\hline$R(p)$ & & & & & \\
LASr (\%) & $0.07(\mathrm{~ns})$ & $0.14(\mathrm{~ns})$ & $-0.01(\mathrm{~ns})$ & $0.17(\mathrm{~ns})$ & $0.16(\mathrm{~ns})$ \\
LASct (\%) & $-0.30(0.002)$ & $0.11(\mathrm{~ns})$ & $-0.37(<0.001)$ & $-0.18(\mathrm{~ns})$ & $0.28(0.004)$ \\
LAScd (\%) & $-0.28(0.004)$ & $0.17(\mathrm{~ns})$ & $-0.31(0.001)$ & $-0.07(\mathrm{~ns})$ & $-0.32(0.001)$ \\
\hline
\end{tabular}

The abbreviations are as in Table 2.

LV GLS values (mean -18.1\%) despite normal LVEF (mean $65.3 \%$ ). Moreover, we observed an association between LV GLS values and the occurrence of LV diastolic dysfunction. The incidence of LV diastolic dysfunction grew higher as the
GLS value was more impaired (from $48.4 \%$ in the group of subjects with LV GLS $<$ the absolute value of $-16.3 \%$ to none in the group of patients with GLS $<$ the absolute value of $-19.9 \%$ ) [5]. Similar findings were reported in a study by 
Galderisi et al. [6], in which reduced LV GLS was noticed in young patients with $\mathrm{AH}$, and its strong dependence on LV diastolic dysfunction and the degree of LV hypertrophy were found.

Dividing our study group depending on the LV GLS showed that patients with lower LV GLS are characterized by decreased values in parameters reflecting reservoir and conduit LA function. Only contractile LA function was comparable between the two groups of patients. Our results agree with previous reports on LAS in AH. Jarasunas et al. noticed impaired values of all LA function, including reservoir, conduit, and pump, in 63 patients with AH. Both LA conduit and reservoir function decreased with an increasing number of parameters showing LV dysfunction, while contractile function did not change [15]. It is worth mentioning that the study by Jarasunas et al. included patients with concomitant paroxysmal atrial fibrillation. This atrial arrhythmia may itself lead to impaired LAS [16-18]. It was also suggested that the LASr value proved to be a strong parameter of LA fibrosis in patients with AF [19].

The correlations of LA reservoir (LASr) and conduit (LAScd) strain with LV GLS values and chosen indices of LV diastolic function imply a complex interplay between LA and LV hemodynamics. It seems logical that an interplay was revealed between LA function represented by both reservoir and conduit LA strain and LV systolic function. LA reservoir strain reflects LA relaxation that lasts throughout the period of LV systole. We can treat LASr as an indicator of LA compliance. LA conduit strain spans early LV filling, so it is closely related to $\mathrm{LV}$ relaxation. LA contractile strain depends on LA myocardial shortening and LV filling pressures, and it is usually impaired in more advanced LA dysfunction [20]. Our results confirmed that LV longitudinal contraction is related to reservoir and conduit LA strain. We did not find any published papers reporting such observations.

Our investigation of relations between LAS and LV diastolic function indices revealed correlations between both conduit (LAScd) and contractile (LASct) strain and $e$ 'avg, $A$, and $E / A$. The pathophysiological mechanisms of LA function can explain these correlations. Conduit LA strain reflects early LV filling; meanwhile, contractile LA strain reflects LV filling during late diastole. Therefore, it seems natural to associate LAScd and LASct with the parameters of both mitral inflow and LV myocardial velocities (especially LAScd with $E$ and $\mathrm{E}^{\prime}$ velocity, LASct with $\mathrm{A}$ and $\mathrm{A}^{\prime}$ velocity, and both LAScd and LASct with $E / A$ ratio) [21]. Olsen et al.'s study of patients with cryptogenic stroke revealed that those with impaired LASr $<28.2 \%$ have more reduced $e$ value and increased $E / e$ ' ratio compared to subjects with $\mathrm{LASr} \geq 28.2 \%$ [22]. No previous studies have confirmed the relations we found among patients with mild hypertension and without any other comorbidities.

Other researchers have reported correlations between LAS and E/e' ratio, which is quite a good parameter for noninvasive estimation of LV filling pressure. In a study of hypertensive patients with confirmed LV diastolic dysfunction, Morris et al. [23] demonstrated that LA function assessed by STE strain was inversely related to the degree of LV diastolic dysfunction and to LV filling pressure (measured by mitral E/e' ratio). Other authors have demonstrated that global LASr provides an even better estimation of LV filling pressure [24]. However, both of these studies concerned patients with more advanced LV dysfunction and higher cardiovascular risk compared to ours.

4.1. Strengths and Limitations. We realize that the methodology and role of STE in the assessment of LA deformation dynamics are still not well established. We assessed strain parameters referring to the actual state of the art. Moreover, the measurements were performed by an experienced echocardiographer, and their reproducibility was checked earlier on 20 randomly selected patients. Additionally, the definition of lower LV GLS and LAS values is still unclear. The expert recommendations speak that a peak GLS in the range of $-20 \%$ can be expected in a healthy person and that is why we decided to use this LV GLS cut-off point. Similarly, we tried to compare our LAS values with these obtained in a large meta-analysis. The strength of our results lays in the homogenous nature of our group-we recruited only hypertensive patients with no concomitant diseases.

\section{Conclusions}

Lower LV and LA strain values occur with a significant percentage of patients with treated, well-controlled, mildstage $\mathrm{AH}$. In particular, reduced values apply to LA reservoir (LASr) and conduit (LAScd) strain parameters. Impaired LA strain is associated with lower LV strain and reduced LV diastolic function parameters, reflecting both the passive and active.

\section{Data Availability}

The datasets used and analyzed during the current study are available from the corresponding author on reasonable request.

\section{Disclosure}

The manuscript has been presented as a preprint according to the following link: https://www.researchsquare.com/ article/rs-35662/v1.

\section{Conflicts of Interest}

The authors declare that they have no conflicts of interest.

\section{Authors' Contributions}

Beata Uziębło-Życzkowska and Paweł Krzesiński contributed to study design; Beata Uziębło-Życzkowska and Paweł Krzesiński performed data collection; Beata UziębłoŻyczkowska and Paweł Krzesiński performed statistical analysis; Beata Uziębło-Życzkowska and Paweł Krzesiński performed data interpretation; Beata Uziębło-Życzkowska and Paweł Krzesiński prepared the manuscript; Beata 
Uziębło-Życzkowska performed literature search; Paweł Krzesiński collected the funds.

\section{Acknowledgments}

The FINEPATH study was supported by the Ministry of Science and Higher Education/Military Institute of Medicine, Warsaw, Poland (Grants nos. 148/WIM and 335/ WIM).

\section{References}

[1] G. Mancia, R. Fagard, K. Narkiewicz et al., "2013 ESH/ESC Guidelines for the management of arterial hypertension," Journal of Hypertension, vol. 31, no. 7, pp. 1281-1357, 2013.

[2] J. T. Wright, J. T. Wright, J. D. Williamson et al., "A randomized trial of intensive versus standard blood-pressure control," The New England Journal of Medicine, vol. 373, no. 22, pp. 2103-2116, 2015.

[3] P. K. Whelton, R. M. Carey, W. S. Aronow et al., "ACC/AHA/ AAPA/ABC/ACPM/AGS/APhA/ASH/ASPC/NMA/PCNA guideline for the prevention, detection, evaluation, and management of high blood pressure in adults: executive summary: a report of the American college of cardiology/ American heart association task force on clinical practice guidelines," Journal of the American College of Cardiology, vol. 71, pp. 2199-2269, 2018;.

[4] B. Uziębło-Życzkowska, P. Krzesiński, G. Gielerak, and A. Skrobowski, "Speckle tracking echocardiography and tissue Doppler imaging reveal beneficial effect of pharmacotherapy in hypertensives with asymptomatic left ventricular dysfunction," Journal of the American Society of Hypertension, vol. 11, pp. 334-342, 2017.

[5] P. Krzesiński, B. Uziębło-Życzkowska, G. Gielerak, A. Stańczyk, M. Kurpaska, and K. Piotrowicz, "Global longitudinal two-dimensional systolic strain is associated with hemodynamic alterations in arterial hypertension," Journal of the American Society of Hypertension: JASH, vol. 9, no. 9, pp. 680-689, 2015.

[6] M. Galderisi, V. S. Lomoriello, A. Santoro et al., "Differences of myocardial systolic deformation and correlates of diastolic function in competitive rowers and young hypertensives: a speckle-tracking echocardiography study," Journal of the American Society of Echocardiography, vol. 23, no. 11, pp. 1190-1198, 201.

[7] S. Mondillo, M. Cameli, M. L. Caputo et al., "Early detection of left atrial strain abnormalities by speckle-tracking in hypertensive and diabetic patients with normal left atrial size," Journal of the American Society of Echocardiography, vol. 24, no. 8, pp. 898-908, 2011.

[8] M. Sahebjam, A. Mazareei, M. Lotfi-Tokaldany, N. Ghaffari, A. Zoroufian, and M. Sheikhfatollahi, "Comparison of left atrial function between hypertensive patients with normal atrial size and normotensive subjects using strain rate imaging technique," Archives of Cardiovascular Imaging, vol. 2, Article ID e16081, 2014.

[9] P. Krzesiński, G. Gielerak, A. Stańczyk et al., "The effect of hemodynamically-guided hypotensive therapy in one-year observation: randomized, prospective and controlled trial (FINEPATH study)," Cardiology Journal, vol. 23, no. 2, pp. 132-140, 2016.

[10] S. F. Nagueh, O. A. Smiseth, C. P. Appleton et al., "Recommendations for the evaluation of left ventricular diastolic function by echocardiography: an update from the American society of echocardiography and the European association of cardiovascular Imaging," Journal of the American Society of Echocardiography, vol. 29, no. 4, pp. 277-314, 2016.

[11] R. M. Lang, L. P. Badano, V. Mor-Avi et al., "Recommendations for cardiac chamber quantification by echocardiography in adults: an update from the American society of echocardiography and the European association of cardiovascular imaging," Journal of the American Society of Echocardiography, vol. 28, no. 1, pp. 1-39, 2015.

[12] V. Mor-Avi, R. M. Lang, L. P. Badano et al., "Current and evolving echocardiographic techniques for the quantitative evaluation of cardiac mechanics: ASE/EAE consensus statement on methodology and indications," Journal of the American Society of Echocardiography, vol. 24, no. 3, pp. 277-313, 2011.

[13] L. P. Badano, T. J. Kolias, D. Muraru et al., "Standardization of left atrial, right ventricular, and right atrial deformation imaging using two-dimensional speckle tracking echocardiography: a consensus document of the EACVI/ASE/Industry task force to standardize deformation imaging," European Heart Journal - Cardiovascular Imaging, vol. 19, no. 6, pp. 591-600, 2018.

[14] F. Pathan, N. D’Elia, M. T. Nolan, T. H. Marwick, and K. Negishi, "Normal ranges of left atrial strain by speckletracking echocardiography: a systematic review and metaanalysis," Journal of the American Society of Echocardiography, vol. 30, no. 1, pp. 59-70, 2017.

[15] J. Jarasunas, A. Aidietis, and S. Aidietiene, "Left atrial strainan early marker of left ventricular diastolic dysfunction in patients with hypertension and paroxysmal atrial fibrillation," Cardiovascular Ultrasound, vol. 16, p. 29, 2018.

[16] S. Yuda, A. Muranaka, and T. Miura, "Clinical implications of left atrial function assessed by speckle tracking echocardiography," Journal of Echocardiography, vol. 14, no. 3, pp. 104-112, 2016.

[17] Y. E. Yoon, I.-Y. Oh, S.-A. Kim et al., "Echocardiographic predictors of progression to persistent or permanent atrial fibrillation in patients with paroxysmal atrial fibrillation (E6P study)," Journal of the American Society of Echocardiography, vol. 28, no. 6, pp. 709-717, 2015.

[18] T. Kojima, M. Kawasaki, R. Tanaka et al., "Left atrial global and regional function in patients with paroxysmal atrial fibrillation has already been impaired before enlargement of left atrium: velocity vector imaging echocardiography study," European Heart Journal-Cardiovascular Imaging, vol. 13, no. 3, pp. 227-234, 2012.

[19] M. Leung, R. Abou, P. J. van Rosendael et al., "Relation of echocardiographic markers of left atrial fibrosis to atrial fibrillation burden," The American Journal of Cardiology, vol. 122, no. 4, pp. 584-591, 2018.

[20] E. Donal, A. Behagel, and D. Feneon, "Value of left atrial strain: a highly promising field of investigation," European Heart Journal - Cardiovascular Imaging, vol. 16, no. 4, pp. 356-357, 2015.

[21] B. D. Hoit, "Left atrial size and function," Journal of the American College of Cardiology, vol. 63, no. 6, pp. 493-505, 2014.

[22] F. J. Olsen, L. M. Christensen, D. W. Krieger et al., "Relationship between left atrial strain, diastolic dysfunction and subclinical atrial fibrillation in patients with cryptogenic stroke: the surprise echo substudy," The International Journal of Cardiovascular Imaging, vol. 36, no. 1, pp. 79-89, 2020.

[23] D. A. Morris, M. Takeuchi, M. Krisper et al., "Normal values and clinical relevance of left atrial myocardial function 
analysed by speckle-tracking echocardiography: multicentre study," European Heart Journal - Cardiovascular Imaging, vol. 16, no. 4, pp. 364-372, 2015.

[24] M. Cameli, S. Sparla, M. Losito et al., "Correlation of left atrial strain and Doppler measurements with invasive measurement of left ventricular end-diastolic pressure in patients stratified for different values of ejection fraction," Echocardiography, vol. 33, no. 3, pp. 398-405, 2016. 